\title{
A Comprehensive Review on Ganoderma lucidum derived Bioactive peptide Ling Zhi-8
}

\author{
Anuja Bhardwaj ${ }^{1}$ and Kshipra Misra ${ }^{1 *}$ \\ Save The Environment, Gurugram, India.
}

\begin{abstract}
Ganoderma lucidum (GL) is a medicinal mushroom which is highly appreciated in Traditional Chinese Medicine and has also been extensively studied in the contemporary world. Scientific investigations have established various medicinal and nutraceutical activities of this medicinal mushroom. The fungus is known to be a repository of numerous bioactive metabolites including terpenoids, polysaccharides, proteins and peptides, nucleotides, fatty acids, steroids, vitamins and minerals. Among these biologically active compounds, the most researched class of compounds are terpenoids and polysaccharides. Consequently, several review articles describing the pharmacological properties of terpenoids and polysaccharides derived from GL are available. Amidst the proteins isolated from GL, Ling Zhi-8 (LZ-8) is the most studied protein. However, no review is available to the best of our knowledge, although there are numerous studies pertaining to the bioactivities of this protein and its recombinant form. The protein LZ-8 has been reported to exhibit immunomodulatory, mitogenic, antitumor, anti-cancer, anti-diabetic and anti-osteoporotic activities. Thus, in this article we intend to summarize the research conducted so far regarding the therapeutic effects and mechanisms of action of LZ-8 protein isolated from GL and also its recombinant form. This shall enable a comprehensive review on the research conducted regarding LZ-8 protein until now.
\end{abstract}

\section{Keywords}

Ganoderma lucidum, fungal immunomodulatory proteins, medicinal mushrooms, Ling Zhi-8, immunomodulation, mitogen.

\section{Introduction}

Since time memorial, medicinal mushrooms have been advantageous to humankind due to their enormous medicinal and nutraceutical benefits. They are recognized as a good source of several biologically active metabolites and hence, are used as health supplements and cosmetics. A medicinal mushroom called as "Ling Zhi”, "Reishi” or Ganoderma lucidum (GL), which is well documented in Traditional Chinese Medicine and Japanese Medicine, has been extensively studied pertaining to its secondary metabolites and their bioactivities. According to the literature survey, GL extracts, fractions and various bioactive compounds isolated from it have demonstrated numerous pharmacological properties including antiangiogenic, anticancer, antiinflammatory, antimicrobial, antioxidative, antitumor, immunomodulatory and neuroprotective activities [1, 2, 3].

The genus Ganoderma consists of approximately 400 different types of bioactive constituents and about 279 secondary bioactive metabolites such as terpenoids, polysaccharides, proteins and peptides, nucleotides, enzymes, fatty acids, steroids, vitamins and minerals have been reported from GL[1, 4]. Among these, the most studied class of metabolites derived from GL are polysaccharides and terpenoids. Consequently, several review articles describing the pharmacological properties of terpenoids and polysaccharides derived from GL are available.

Nevertheless, recently studies on peptides isolated from GL has commenced by several researcher groups. Amidst the proteins isolated from GL, Ling Zhi-8 (LZ-8) is the most studied protein. However, no review is available to the best of our knowledge, although there are numerous studies pertaining to the bioactivities of this protein and its recombinant form. The protein LZ-8 has been reported to exhibit immunomodulatory, mitogenic, anti-tumor, anticancer, anti-diabetic and anti-osteoporotic activities [2]. 
Thus, in this article we intend to summarize the research conducted so far regarding the therapeutic effects and mechanisms of action of LZ-8 protein isolated from GL and also its recombinant form. This shall enable a comprehensive review on the research conducted regarding LZ-8 protein until now.

\section{Ling Zhi-8 (Lz-8)}

Fungi are considered a rich source of proteins [5] and consist of over $30 \%$ of the mushroom dry weight [6]. At present, proteins namely, Ling Zhi-8 (LZ-8), Ganodermin, LZP-1, LZP-2 and LZP-3 and several others have been isolated from Ganoderma lucidum (GL) and characterized using electrophoretic and chromatographic techniques [7]. In the sections below, we have described the various biological activities of GL derived protein LZ-8 and associated mechanisms.

\section{Structural and Physicochemical Characteristics of Ling Zhi-8}

Ling Zhi-8 (LZ-8) or FIP-glu belongs to the family of fungal immunomodulatory protein (FIP).

To date, LZ-8 (FIP-glu), FIP-gts, FIP-fve, FIP-vvo, FIP-gja, FIP-gmi, FIP-gsi and FIP-tve have been identified from Ganoderma lucidum, Ganoderma tsugae, Flammulina velutipes, Volvariella volvacea, Ganoderma japoncium, Ganoderma microsporum, Ganoderma sinensis and Trametes versicolor[8].These FIPs are small molecule proteins with wide-ranging bioactivities such as anti-allergy, antitumor, ability to stimulate immune cells to produce a variety of cytokines, etc. [8, 9]. Ling Zhi-8 (LZ-8) is known to be the first protein isolated from the mycelium culture of Ganoderma lucidum by Kino et al [10]. The protein was purified by using two chromatographic techniques sequentially. At first, by gel filtration chromatography using Sephadex G-75 column (5 X $100 \mathrm{~cm})$, previously equilibrated with $10 \mathrm{mM}$ Tris-HCl buffer (pH 8.0). Afterwards, ion-exchange chromatography was employed by absorbing the most active fraction onto a DEAE-Sephadex A25 column $(2.6 \times 40 \mathrm{~cm})$, previously equilibrated with $10 \mathrm{mM}$ Tris-HCl buffer (pH 8.0). The authors reported the molecular weight of the purified protein, i.e., LZ-8 to be $13 \mathrm{kDa}$ as determined by Tricine-sodium dodecyl surface-PAGE electrophoresis with an isoelectric point of 4.4. LZ-8 was found to contain only two sugars- mannose and hexosamine which constituted $0.6 \%$ of its sugar content. It comprised of relatively large amounts of asparagine (or aspartic acid) and valine. Half-cysteine, histidine, methionine, and hydroxyproline were not detected in the protein [10].

The native form of LZ-8 has a molecular mass of $24 \mathrm{kDa}$ is a homodimer of the LZ-8 polypeptide. Each polypeptide chain consists of 110 amino acid residues, and has molecular mass of 12,420 Da which includes the amino-end acetyl group also. Within the structure of LZ-8, there is no consensus sequence Asn-X-Ser or Thr as an attachment site for an Asn-linked oligosaccharide chain. This corresponds to the very low carbohydrate content of LZ-8 as shown previously [10].
Furthermore, the LZ-8 polypeptide chain exhibits significant similarity both in its sequence and in its predicted secondary structure to the variable region of immunoglobulin heavy chain $\left(\mathrm{I}_{\mathrm{g}} \mathrm{V}_{\mathrm{H}}\right)$ [11].

\section{Mitogenic activity of Ling Zhi-8}

Recent findings have revealed that a homodimeric structure is necessary for the immunomodulatory ability of FIPs. Dimerization is the key process through which FIPs bind to their cell surface receptors and exert their activity. This fact has been revealed by a recent study, wherein a structure-based multiple alignment of LZ-8 and an FIP from Volvariella volvacea was performed. During this study, the electrostatic potential of their protein surfaces was compared and a model summarizing the unique electrostatic interaction in LZ-8 dimerization was developed. The results highlighted the fact that dimerization of native LZ-8 or rLZ-8 plays a crucial role in inducing the expression of IL-2. The interleukin, IL-2 is responsible for the regulation of certain autoimmune processes and in the generation and function of regulatory Tcells [9].

Previously also, LZ-8 has been reported to demonstrate mitogenic activity on human peripheral blood lymphocytes (PBL) by acting as a potent T-cell activator and mediating its effects byupregulating IL-2 and intercellular adhesion molecule-1 (ICAM-1) expression along with an increased production of IFN- $\gamma \mathrm{TNF} \alpha$, and IL- $\beta$ molecules, which are known to be associated with regulation of ICAM-1 expression [12].

The intercellular adhesion molecule- 1 is a transmembrane glycoprotein belonging to the immunoglobulin (Ig) superfamily. It is expressed by several cell types including endothelial cells, fibroblasts, andleucocytes. ICAM-1 plays crucial roles in adhesion of cells, trans-endothelial migration of leucocytes to sites of inflammation and lymphocytes activation [13]. Further, Bao et al., performed electrostatic potential and virtual amino acid mutation analyses which indicated that L10, W12 and D45 are the key amino acid residues responsible for the high immunomodulatory activity of LZ-8. Hence, structural and physicochemical properties are major factors affecting the biological activities of FIPs [9].

It was in the year 1989, when Kino et al., first examined the hemagglutination activity of LZ-8 using human and sheep red blood cells (RBCs). The assay revealed that LZ-8 could not agglutinate human RBCs, but, the protein agglutinated sheep RBCs. The difference in the hemagglutination activity of LZ8 towards human and sheep RBS was unexplainable by the authors. Furthermore, their study confirmed that LZ-8 is not a lectin.

The same group also studied the mitogenic activity and immunomodulatory activity of LZ-8. The mitogenic activity was assessed by determining effect of LZ-8 on $\left[{ }^{3} \mathrm{H}\right]$ thymidine uptake by cultured spleen cells alone or in the presence of 
LPS or Con A. The results indicated an additive effect of LZ-8 and LPS at low concentrations (0.13-3.13 microgram $/ \mathrm{ml}$ ) on $\left[{ }^{3} \mathrm{H}\right]$ thymidine uptake and hence, it was inferred that the target cell of LZ-8 might be different from that of LPS; which is known to activate B-lymphocytes [10].LZ-8 has also shown its mitogenic activity towardshuman peripheral mononuclear cell and mouse splenocytes. However, the mitogenic effect of LZ-8 obligated the presence of monocytes [14].

\section{Immunomodulatory activity of Ling Zhi-8}

The immunomodulatory activity of LZ-8 was first investigated by determining its effect on systemic anaphylaxis in Carworth Farm Webster (CFW) mice. Treatment of mice with LZ-8 suppressed BSA-induced anaphylaxis completely. Moreover, the treatment with LZ-8 (twice weekly) of CFW mice before sensitization with BSA via any systemic route, prevented anaphylaxis. This suggested that the LZ-8 is an immunosuppressant which acts by inhibiting antibody production.

The immunomodulatory activity of LZ-8 was further confirmed by Arthus reaction. In the Arthus reaction, the extent of immediate reaction at the intradermal site of antigen administration depends on the quantity of existing antibodies producing following sensitization. A reduction in the number of Arthus reaction-positive mice was observed from 90 to $40 \%$. This led to the conclusion that in vivo, LZ-8 reduces antibody production but does not block it, totally [10]. Another group also observed that the immunosuppressive activities of LZ-8 caused by the blocking of antigen-specific antibody production. In this study, the in vivo effect of LZ-8 on antibody production using the hepatitis B surface antigen (HBs Ag) in mice was conducted. LZ-8 exhibited mitogenic activity in vitro towards spleen cells of C57BL/10 (B10) and C57BL/10BR (B10BR). The intraperitoneal administration of LZ-8 twice weekly into the mice (8 and $12 \mathrm{mg} / \mathrm{kg}$ ) significantly suppressed antibody production to $\mathrm{HBs} \mathrm{Ag}$ (83.3-96.8\%), whereas, B10 and B10BR mice produced antiHBs Ag antibody by the twice sensitization of the antigen [15].

\section{Anti-diabetic activity of Ling Zhi-8}

Diabetes is a disease that involves immune responses. LZ-8 has also demonstrated an important role in preventing insulitis as observed in non-obese diabetic (NOD) mouse through its immunomodulatory action. The protein was found to reduce lymphocyte infiltration, augmented antibody detection of insulin in beta cells and reduced plasma glucose concentration in NOD mice. T cell subset population analysis revealed that LZ-8 regulatedsubsets of immune cells [16]. Furthermore, the immunosuppressive effect of LZ-8 has also been demonstrated in vitro in a human mixed lymphocyte culture (MLC) performed in the absence of monocytes, using purified T-cells and Epstein Bar Virus (EBV)-transformed allogeneic B-cells. This group alsostudiedthe plausible suppressive effects of LZ-8 in two different models of allogeneic tissue transplantation- allografted mouse skin model and transplanted allogeneic pancreatic rat islets. Administration of LZ-8 in allografted mouse skin model caused an increase in survival time and had a substantial effect on cellular immunity. LZ-8 facilitated delay in the rejection process in the transplanted allografted pancreatic rat islets model [14].

\section{Hepatoprotective activity of Ling Zhi-8}

Besides, mitogenic and immunomodulatory effects, LZ-8 has also demonstrated hepatoprotective properties also, against tetrachloride carbon $\left(\mathrm{CCl}_{4}\right)$-stimulated hepatic injury when administration orally to rats [17]. Moreover, a study has revealed potent wound healing activityof LZ-8 against the electrosurgical induced liver injury. As suggested by the authors, the effect was due to the inhibition of NF B and caspase-3 expressions by LZ-8. NF B is reported to be an important link between hepatic injury, fibrosis, and even hepatocellular carcinoma[18].

\section{Anti-inflammatory activity of Ling Zhi-8}

The protein LZ-8 has shown its anti-inflammatory activity for modulating in vitro immune responses involved in neural inflammation in murine microglial BV-2 cells. BV-2 is a type of microglial cell derived from C57/BL6 murine. In this study, LZ-8 regulated the LPS-activated immune responses of BV-2 cells by reducing the production of proinflammatory mediators, comprising NO, PGE2, IL-6 and, expression of iNOS and COX-2, via suppression of TLR4mediated NF-kB signaling [19].

\section{Anticancer activity of Ling Zhi-8}

The protein, LZ-8 exhibits anti-cancer progression and metastasis activity. Recently, it has been reported that LZ-8 produced changes in the proteomic profile (21 proteins) of tumor lesions. In particular, three heat shock proteins (HSPs), namely, HSP60, 70 and 90, were significantly downregulated in tumor lesions of Lewis lung carcinoma 1 (LLC1)-bearing mouse administered with LZ-8 in comparison to control (PBS; phosphate buffered saline). Furthermore, LZ-8 effectively inhibited cell migration and decreased cell viability of LLC1 cells[20]. Earlier, the same group had demonstrated that recombinant LZ-8 reduces the tumor progression in lung cancer LLC1 cell-bearing mouse[21].

\section{Recombinant LZ-8 Protein(rLZ-8)}

It became evident from various researches that LZ-8 has potential immunomodulatory and mitogenic activities. However, the isolation and purification of LZ-8 from Ganoderma lucidum mycelia was a constraint in the research pertaining to LZ-8. This is because isolation of the native LZ8 from Ganoderma lucidum mycelia and its purification is an expensive and laborious process [22, 23]. Therefore, production of recombinant LZ-8 at high-level was opted to circumvent these complications. Expression vectors such as E. coli[22] and Pichia pastoris[24] have been used to generate rLZ-8 with better performance in terms of immunomodulatory and mitogenic activities. Recombinant protein production is a beneficialmean to avoid 
contamination from cellular proteins and facilitates continuous cultures for downstream processing [23].

The production of LZ-8 into several expression vectors, subsequently led to numerous scientific studies using recombinant LZ-8 (rLZ-8) for elucidating the potential role under several pathological conditions and better understanding of various pharmacological activities possessed by rLZ-8; which has demonstrated similar biological effects as its native form, i.e., LZ-8. Some of these conducted using rLZ-8 are detailed in the following sections.

\section{Antitumor activity of rLZ-8}

Wu et al in 2011, elucidated that rLZ-8 increased G1 arrest and, activated p53 and p21 expressions in A549 human lung cancer cells. Moreover, administration of rLZ-8 in mice transplanted with Lewis lung carcinoma cells resulted in ribosomal stress via inhibition of precursor ribosomal RNA synthesis and reduced polysome formation in A549 cells. This further caused an increasing binding of ribosomal protein S7 to MDM2 and a decreased interaction between MDM2 and p53. It is well known that various stress can activate p53 through disruption of p53-MDM2 interaction. In response to ribosomal stress, several RPs, including L5, L11, L23 and S7, can bind to MDM2 and block MDM2-mediated p53 ubiquitination and degradation. This results in p53dependent cell cycle arrest and/or apoptosis. Thus, the current study highlighted the antitumor activity of rLZ-8, which inhibited lung cancer growth in vitro and in vivo[25].

In another study, the underlying mechanism for tumor metastasis suppression activity and increased survival rate in Lewis lung carcinoma cell-bearing mice of rLZ-8 was explained. Mechanistically, rLZ-8 induced focal adhesion kinase (FAK) inactivation which downregulated Slug by enhancing ubiquitination proteasome pathway (UPP)mediated degradation of Slug. Consequently, E-cadherin expression was enhanced and cancer cell mobility was repressed. Nevertheless, MDM2-shRNA obliterated rLZ-8enhanced Slug degradation. rLZ-8 may be useful as a chemotherapeutic agent for treating lung cancer.EMT process is an important process in tumor metastasis and Slug is a transcription factor that represses E-cadherin transcription and is a crucial event in EMT and tumor metastasis[21].

In the following year, the same group established that rLZ-8 induced cell cycle arrest and apoptosis by downregulating the expression of wild-type and mutated epidermal growth factor receptor (EGFR) and inhibiting EGFR downstream effectors, AKT and ERK-1/2 in lung cancer cells. The progression of malignant lung tumor is associated with activation of mutation in EGFR. During the study, it was also observed that binding of rLZ-8 to EGFR resulted in induction of EGFR autophosphorylation and triggered ubiquitination by stimulating EGFR/Cbl complexes formation. Thereby, causing degradation of EGFR [26]. Later, in vitro growth arrest and apoptotic function of rLZ-8 (FIP-glu) and FIP-
SN15 in human glioblastoma U-251 MG cells was also reported.FIP-SN15 is the recombinant DNA sequence generated by DNA shuffling technology between FIP-glu and FIP-gsi [8].

Progressively, the adjuvanticity of LZ-8 for HER-2/neu DNA vaccine against $\mathrm{p} 185^{\text {neu }}$ expressing tumor murine bladder tumor cell line (MBT-2) in mice has also been demonstrated. HER-2/neu $\left(\mathrm{p} 185^{\mathrm{neu}}\right)$ is a transmembrane tyrosine kinase receptor and have been associated with tumor progression in certain neoplasms [27]. In this study, rLZ-8 stimulated mouse bone marrow-derived dendritic cells (DCs) via toll-like receptor 4 (TLR4) and augmented the ability of DCs to induce antigen-specific T-cell activation in vitro and in vivo (in a subunit vaccine model). The antitumor effect of DNA vaccine against MBT-2 tumor in mice was improved with cotreatment with rLZ-8. The mechanism for this amplified antitumor activity was due to the enhancement of vaccine-induced Thelper cells (Th1) and cytotoxic T lymphocytes (CTL) responses. Thus, this work emphasized an important application of rLZ-8 as a promising adjuvant for enhancing the therapeutic efficacy of DNA vaccines against tumors [28].

\section{Antidiabetic activity of $r L Z-8$}

In a recent study, anti-diabetic activity of rLZ-8 instreptozocin (STZ)-induced diabetic rats was described and the underlying mechanism was explained as well. Administration of rLZ-8 into STZ-induced diabetic rats for 3 months alleviated the clinical symptoms of type 1 diabetes (T1D) and dose-dependently diminished blood glucose, blood lipid and hemoglobin A1c (HbA1c) levels. There was a temporary but prominent elevation in insulin levelswhich protected against STZ-induced pancreatic tissue injury. Moreover, rLZ-8 significantly lowered TNF- $\alpha$ and IL-1 $\beta$ levels and raised IL-10 levels in serum and pancreas. Furthermore, rLZ-8 treatment resulted in substantial increment in regulatory T-cells (Tregs) number and upregulated Fork head box P3 (Foxp3) expression thereby, reinstating the balance between inflammatory and antiinflammatory cytokines[29]. The Tregs have been proven to maintain immune homeostasis and play a critical role in tissue regeneration after injury[30].

Similar effects of rLZ-8 were observed during a study, wherein, efficacy of rLZ-8 in curbing pulmonary inflammation was observed inOVA-induced asthmatic mice. In this study also, rLZ-8 remarkably downregulated Th17 cells and upregulated Foxp3+ regulatory $\mathrm{T}$ (Treg) cells. In bronchoalveolar lavage fluid (BALF), IL-17A level was decreased whereas, IL-10 level was increased. At the same time in the lung tissue, RORyt mRNA expression was diminished and Foxp3 mRNA level improved. Both, in murine lung tissue as well as cultured T-cells, rLZ-8 repressed signaling pathways of STAT3 as well as NF- $\mathrm{KB}$ (P100/P52). Thus, rLZ-8 reduced pulmonary inflammation[31]. 


\section{Therapeutic effects of rLZ-8 against tumor chemotherapy induced-neutropenia and leukemia}

The recombinant protein has also been evaluated for its protective effects against cyclophosphamide inducedneutropenia [32] and leukopenia [33] in order to elucidate its potential to prevention of neutropenia and leukemia during tumor chemotherapy.Neutropenia is a clinical condition with an abnormally low levels of neutrophils in the blood and is associated with chemotherapy. In the study led by Lei et al., it was demonstrated that rLZ-8 promoted the differentiation of bone marrow hematopoietic stem cells (HSCs) into granulocytes by binding to colony stimulating factor 1 receptor. It also stimulated the mobilization of HSCs and the release of neutrophils from the bone marrow to peripheral blood by regulating the CXCR4-SDF1 axis [32].

The study pertaining to the therapeutic effect of rLZ-8 on mouse models of cyclophosphamide-induced leukopenia wasdesigned in two ways- single-phase and multi-phase administration methods. In both the models,rLZ-8treatment resulted in increment in the levels of neutrophils, lymphocytes and monocytes. Moreover, it elevated CD4+ Tcells percentage and also raised secretion of IL-3 and IL-4. Conclusively, the study established the fact that treatment with rLZ-8 was advantageous in improving immune dysfunction and immune system imbalance[33].

\section{Anti-osteoporotic activity of $r \mathrm{LZ}-8$}

Current studies have also established the potential role of rLZ-8 in improving the clinical and pathological conditions associated with osteoporosis.In a study the efficacy of rLZ-8 was examined on osteoclast in vitro and bone resorption in vivo. Under in vitro conditions, receptor activator of nuclear factor kappa-B (RANK) ligand induced RAW 264.7 cells were allowed to differentiate into osteoclastic cells. These cells were then treated with various doses of rLZ-8 for seven days and differences in osteoclastic differentiation, apoptosis rate and gene expression were measured with respect to the control. Three dimensional-structured illumination microscopy analysis revealed that rLZ-8 entered and got accumulated gradually into the cytoplasm of RAW264.7 cells but very little quantity escaped into the nucleus.

Additionally, under in vivo conditions, retinoic acid was administered to female rats for 14 consecutive daysto develop osteopenia changes. Simultaneously, different doses of rLZ-8 were administered to rats treated with retinoic acid to observe changes of bone mineral density, biochemical parameters and organ weight ratio. The results of the study suggested that rLZ-8 inhibited osteoclastic differentiation and promoted osteoclastic apoptosis by regulating RANK- tumor necrosis factor receptor-associated factor 6 (TRAF6) - c-Jun Nterminal kinase (JNK) signaling pathway. Thus, administration with rLZ-8 reversed loss of bone mass and improved ALP activity in osteoporotic rats. Low-to highdose rLZ-8 treatments displayed little toxic effects on rat organs. Overall, the data advocated anti-osteoporotic effect of rLZ-8[34].
Furthermore, Yang et al, have also reported therapeutic effects of rLZ-8 in glucocorticoids-induced osteoporosis (GIOP) rat model. The glucocorticoid dexamethasone (DEX) was injected intramuscularly into Wistar rats. During the study, it was found that rLZ-8 could prevent bone loss and improved structural deterioration in femurs of GIOP rats. This observation was confirmed by H\&E staining performed for analyzing the amount and morphology of trabeculae. Furthermore, Western blot analysis of OPG and RANKL expression levels in femurs showed that rLZ-8 could increase OPG/RANKL ratio which consequently delayed the process of osteoclastogenesis. Thus, the results of this study emphasized on the fact that rLZ-8 could be developed as an anti-osteoporosis drug for both prevention and treatment of osteoporosis[35].

\section{Conclusion}

Ling Zhi-8 is a valuable fungal immunomodulatory protein both in its native (LZ-8) and recombinant form (rLZ-8). It has already established its potential role in ameliorating several disease and clinical conditions. Furthermore, the protein has demonstrated no or minimal toxicity as per reports until now. It is therefore feasible to employ LZ-8 and/or rLZ-8 as a potent drug for the prevention and treatment of diseases, especially those involving immunological responses such as cancer, autoimmune diseases, osteoporosis, diabetes, etc.

\section{References}

1. Ahmad MF. Ganoderma lucidum: a rational pharmacological approach to surmount cancer. Journal of Ethnopharmacology. 2020 Oct 5;260:113047.

2. Bhardwaj A, Sharma P, Mishra J, Misra K. Lingzhi or reishi medicinal mushroom, Ganoderma lucidum (Agaricomycetes), mycelium aqueous extract modulates high-altitude-induced stress. International Journal of Medicinal Mushrooms. 2019;21(5):443-458.

3. Lu J, He R, Sun P, Zhang F, Linhardt RJ, Zhang A. Molecular mechanisms of bioactive polysaccharides from Ganoderma lucidum (Lingzhi), a review. International journal of biological macromolecules. 2020 May 1;150:765-74.

4. Oludemi T, Barros L, Prieto MA, Heleno SA, Barreiro MF, Ferreira IC. Extraction of triterpenoids and phenolic compounds from Ganoderma lucidum: optimization study using the response surface methodology. Food \& function. 2018;9(1):209-226.

5. Sinha SK, Upadhyay TK, Sharma SK. Nutritionalmedicinal profile and quality categorization of fresh white button mushroom. Biointerface Res. Appl. Chem. 2021;11:8669-85.

6. Dai, R.; Liu, M.; Nik Nabil, W.N.; Xi, Z.; Xu, H. Mycomedicine: A Unique Class of Natural Products with Potent Anti-tumour Bioactivities. Molecules 2021, 26, 1113. DOI: https://doi. org/10.3390/ molecules 26041113

7. Bhardwaj A, Misra K. Ganoderma sp.: The Royal Mushroom for High-Altitude Ailments. Management of High Altitude Pathophysiology. 2018 Jan 1:115-52. 
8. Cong WR, Xu H, Liu Y, Li QZ, Li W, Zhou XW. Production and functional characterization of a novel fungal immunomodulatory protein FIP-SN15 shuffled from two genes of Ganoderma species. Applied microbiology and biotechnology. 2014 Jul;98(13):596775.

9. Bao DP, Bai R, Gao YN, Wu YY, Wang Y. Computational Insights into the Molecular Mechanism of the High Immunomodulatory Activity of LZ-8 Protein Isolated from the Lingzhi or Reishi Medicinal Mushroom Ganoderma lucidum (Agaricomycetes). Int J Med Mushrooms. 2018;20(6):537-548.

10. Kino K, Yamashita A, Yamaoka K, Watanabe J, Tanaka S, Ko K, Shimizu K, Tsunoo H. Isolation and characterization of a new immunomodulatory protein, ling zhi-8 (LZ-8), from Ganoderma lucidum. Journal of Biological Chemistry. 1989 Jan 5;264(1):472-478.

11. Tanaka S, Ko K, Kino K, Tsuchiya K, Yamashita A, Murasugi A, Sakuma S, Tsunoo H. Complete amino acid sequence of an immunomodulatory protein, ling zhi-8 (LZ-8): an immunomodulator from a fungus, Ganoderma lucidum, having similarity to immunoglobulin variable regions. Journal of Biological Chemistry. 1989 Oct 5;264(28):16372-7.

12. Haak-Frendscho M, Kino K, Sone T, Jardieu P. Ling Zhi-8: a novel $\mathrm{T}$ cell mitogen induces cytokine production and upregulation of ICAM-1 expression. Cellular immunology. 1993 Aug 1;150(1):101-13.

13. Figenschau SL, Knutsen E, Urbarova I, Fenton C, Elston B, Perander M, Mortensen ES, Fenton KA. ICAM1 expression is induced by proinflammatory cytokines and associated with TLS formation in aggressive breast cancer subtypes. Scientific reports. 2018 Aug 6;8(1):1-2.

14. van der Hem LG, van der Vliet JA, Bocken CF, Kino K, Hoitsma AJ, Tax WJ. Ling Zhi-8: studies of a new immunomodulating agent. Transplantation. 1995;60:438-43.

15. Kohsuke K, Toshio S, Watanabe J, Yamashita A, Tsuboi H, Miyajima H, Tsunoo H. Immunomodulator, LZ-8, prevents antibody production in mice. International journal of immunopharmacology. 1991 Jan 1;13(8):1109-15.

16. Kino K, Mizumoto K, Sone T, Yamaji T, Watanabe J, Yamashita A, Yamaoka K, Shimizu K, Ko K, Tsunoo H. An immunomodulating protein, Ling Zhi-8 (LZ-8) prevents insulitis in non-obese diabetic mice. Diabetologia. 1990 Dec;33(12):713-8.

17. van der Hem LG, van der Vliet JA, Bocken CF, Kino K, Hoitsma AJ, Tax WJ. Ling Zhi-8: studies of a new immunomodulating agent. Transplantation. 1995;60:438-43.).

18. Yang CY, Chuang LT, Huang WC, Hou CW, Chen DC, Jeng KC, Kao TY. Preventive effects of borage oil and Ling-Zhi-8 protein on carbon tetrachloride-induced acute hepatic toxicity in rats. Current Topics in Nutraceutical Research. 2014Aug 1;12(3):91-100.

19. Lin HJ, Chang YS, Lin LH, Haung CF, Wu CY, Ou KL. An Immunomodulatory Protein (Ling Zhi-8) from a Ganoderma lucidum Induced Acceleration of Wound Healing in Rat Liver Tissues after Monopolar Electrosurgery. Evidence-based Complementary and Alternative Medicine: Ecam. 2014 May 5;2014:916531.

20. Chen SJ, Lin HH, Huang WC, Tsai PJ, Chen WP, Chen DC, Chuang LT. Ling-Zhi-8 protein (LZ-8) suppresses the production of pro-inflammatory mediators in murine microglial BV-2 cells. Food and Agricultural Immunology. 2017 Nov 2;28(6):1393-407.

21. Lin TY, Hua WJ, Yeh H, Tseng AJ. Functional proteomic analysis reveals that fungal immunomodulatory protein reduced expressions of heat shock proteins correlates to apoptosis in lung cancer cells. Phytomedicine. 2021:153-384.

22. Lin TY, Hsu HY. Ling Zhi-8 reduces lung cancer mobility and metastasis through disruption of focal adhesion and induction of MDM2-mediated Slug degradation. Cancer letters. 2016 Jun 1;375(2):340-8.

23. Ye Boping, Wang Fan, Liang Yixin, Jin Guoqian, Wu Wutong. Prokaryotic expressing of LZ-8 gene in E-coli Pharmaceutical Biotechnology. 2002 ;9(1):21-23.

24. Yeh CM, Yeh CK, Hsu XY, Luo QM, Lin MY. Extracellular expression of a functional recombinant Ganoderma lucidium immunomodulatory protein by Bacillus subtilis and Lactococcus lactis. Applied and environmental microbiology. 2008 Feb 15;74(4):103949.

25. Xue Q, Ding Y, Shang C, Jiang C, Zhao M. Functional expression of LZ-8, a fungal immunomodulatory protein from Ganoderma lucidium in Pichia pastoris. The Journal of general and applied microbiology. 2008;54(6):393-8.

26. Wu CT, Lin TY, Hsu HY, Sheu F, Ho CM, Chen EI. Ling Zhi-8 mediates p53-dependent growth arrest of lung cancer cells proliferation via the ribosomal protein S7-MDM2-p53 pathway. Carcinogenesis. 2011 Dec 1;32(12):1890-6.

27. Lin TY, Hsu HY, Sun WH, Wu TH, Tsao SM. Induction of Cbl-dependent epidermal growth factor receptor degradation in Ling Zhi-8 suppressed lung cancer. International journal of cancer. 2017 Jun 1;140(11):2596-607.

28. Cong WR, Xu H, Liu Y, Li QZ, Li W, Zhou XW. Production and functional characterization of a novel fungal immunomodulatory protein FIP-SN15 shuffled from two genes of Ganoderma species. Applied microbiology and biotechnology. 2014 Jul;98(13):596775. 
29. Osman I, Scher HI, Drobnjak M, Verbel D, Morris M, Agus D, Ross JS, Cordon-Cardo C. HER-2/neu (p185neu) protein expression in the natural or treated history of prostate cancer. Clinical Cancer Research. 2001 Sep 1;7(9):2643-7.

30. Lin CC, Yu YL, Shih CC, Liu KJ, Ou KL, Hong LZ, Chen JD, Chu CL. A Novel Adjuvant Ling Zhi-8 Enhances the Efficacy of DNA Cancer Vaccine by Activating Dendritic Cells. Cancer immunology, immunotherapy: CII. 2011 Jul;60(7):1019-27.

31. Xiao H, Fang Z, He X, Ding P, Cao Y, Chan S, Hou S, Liang J. Recombinant ling zhi-8 enhances Tregs function to restore glycemic control in streptozocininduced diabetic rats. Journal of Pharmacy and Pharmacology. 2020 Dec;72(12):1946-55.

32. Koliesnik IO, Andreas N, Thuy A, Sreekantapuram S, Haenold R, Weih F. Alternative NF-kB signaling controls peripheral homeostasis and function of regulatory $\mathrm{T}$ cells. Immunobiology. 2019 Sep 1;224(5):687-96.

33. Liu H, Qiu F, Wang Y, Liang F, Liang J, Lin C, Liang J, Gong B, Chan S, De Zhang Z, Lai X. A recombinant protein rLZ-8, originally extracted from Ganoderma lucidum, ameliorates OVA-induced lung inflammation by regulating Th17/Treg balance. Journal of Leukocyte Biology. 2020 Aug;108(2):531-45.

34. Lei X, Zhi C, Huang W, Sun X, Gao W, Yin X, Zhang X, Liang C, Zhang H, Sun F. Recombinant Ganoderma lucidum Immunomodulatory Protein Improves the Treatment for Chemotherapy-Induced Neutropenia. Frontiers in pharmacology. 2020;11:956-.

35. Zhou H, Sun F, Li H, Zhang S, Liu Z, Pei J, Liang C. Effect of recombinant Ganoderma lucidum immunoregulatory protein on cyclophosphamideinduced leukopenia in mice. Immunopharmacology and immunotoxicology. 2013 Jun 1;35(3):426-33.

36. Ruan L, Jiang N, Guo F, Xu H, Zhang J, Sun J. The antiresoptive effects of recombinant Lingzhi-8 protein against retinoic acid-induced osteopenia. European Journal of Pharmacology. 2019 Sep 19;863:172669-.

37. Yang Y, Yu T, Tang H, Ren Z, Li Q, Jia J, Chen H, Fu J, Ding S, Hao Q, Xu D. Ganoderma lucidum Immune Modulator Protein rLZ-8 Could Prevent and Reverse Bone Loss in Glucocorticoids-Induced Osteoporosis Rat Model. Frontiers in Pharmacology. 2020 May 19;11:731. 